\title{
A homing suppression gene drive with multiplexed gRNAs maintains high drive conversion efficiency and avoids functional resistance alleles
}

Emily Yang ${ }^{1,2}$, Matthew Metzloff ${ }^{1,2}$, Anna M. Langmüller ${ }^{1,3,4}$, Andrew G. Clark ${ }^{1,2}$, Philipp W. Messer $^{1}$, Jackson Champer ${ }^{1,2,5^{*}}$

${ }^{1}$ Department of Computational Biology, Cornell University, Ithaca, NY 14853

${ }^{2}$ Department of Molecular Biology and Genetics, Cornell University, Ithaca, NY 14853

${ }^{3}$ Institut für Populationsgenetik, Vetmeduni Vienna, Veterinärplatz 1, 1210 Wien, Austria

${ }^{4}$ Vienna Graduate School of Population Genetics, 1210 Wien, Austria

${ }^{5}$ Current Address: Center for Bioinformatics, School of Life Sciences, Peking-Tsinghua Center for Life Sciences, Peking University, Beijing, China 100871

*Corresponding author: JC (jchamper@pku.edu.cn)

\begin{abstract}
Gene drives are engineered alleles that can bias inheritance in their favor, allowing them to spread throughout a population. They could potentially be used to modify or suppress pest populations, such as mosquitoes that spread diseases. CRISPR/Cas9 homing drives, which copy themselves by homology-directed repair in drive/wild-type heterozygotes, are a powerful form of gene drive, but they are vulnerable to resistance alleles that preserve the function of their target gene. Such resistance alleles can prevent successful population suppression. Here, we constructed a homing suppression drive in Drosophila melanogaster that utilized multiplexed gRNAs to inhibit the formation of functional resistance alleles in its female fertility target gene. The gRNA target sites were placed close together, preventing reduction in drive conversion efficiency. The construct reached a moderate equilibrium frequency in cage populations without apparent formation of resistance alleles. However, a moderate fitness cost prevented suppression of the cage population. Nevertheless, our results experimentally demonstrate the viability of the multiplexed gRNAs strategy in homing type suppression gene drives.
\end{abstract}




\section{INTRODUCTION}

At the frontier of pest and disease vector control, gene drives hold the potential to influence large, wild populations. These engineered genetic elements have the ability to spread quickly by biasing inheritance in their favor, allowing for the manipulation of population sizes or traits such as disease transmission ${ }^{1-5}$.

Gene drives can act through many mechanisms and include both engineered and naturally occurring forms ${ }^{6}$. For engineered homing drives, the CRISPR/Cas 9 system has been widely used to create gene drive constructs in many organisms, including yeast ${ }^{7-10}$, flies ${ }^{11-25}$, mosquitoes ${ }^{26-35}$, and mice ${ }^{36}$. The homing mechanism converts an organism heterozygous of the drive into a homozygote in the germline, and the drive is thus transmitted to offspring at a rate above $50 \%$. These drives contain a Cas9 endonuclease, which cleaves a target sequence, and at least one guide RNA (gRNA), which directs Cas9 to the cleavage location. The resulting DNA break can be repaired by homology-directed repair (HDR) using the drive allele as a template, thereby copying the drive into the wild-type chromosome.

However, a major obstacle that impedes drive efficiency is the alternative DNA repair method of end-joining, which does not use a homologous template and often alters the target sequence, preventing further recognition by the gRNA/Cas9 system. Such mutations are therefore considered resistance alleles and can form at high rates in the germline as well as in the embryo due to cleavage activity from maternally deposited Cas9 and gRNA $11,17,20-22,24,25,27,28,34,35$. Resistance alleles that disrupt the function of the target gene by causing frameshifts or otherwise sufficiently changing the amino acid sequence tend to be more common in almost all gene drive designs, and we call them "r2" alleles. By contrast, "r1" alleles" preserve gene function and are therefore particularly detrimental to gene drives. If the drive allele imposes a greater fitness cost than the resistance allele, which is usually the case for $\mathrm{r} 1$ alleles in most drives that target native genes, then the resistance alleles will outcompete the drive and thwart its potential to modify or suppress the population ${ }^{20,24,26,37-39}$.

While modification drives aim to genetically alter a population, for instance by spreading a specific gene variant or genetic cargo, the goal of suppression drives is to ultimately reduce and potentially even eliminate a population, usually by disrupting the gene target of the drive. For example, such a drive could cleave and be copied into a gene with a recessive knockout phenotype that affects viability or fecundity. As the drive increases in frequency in the population (via heterozygotes, which remain fertile and viable ${ }^{40}$ ), the proportion of sterile or nonviable individuals will increase, thereby reducing population size. Even if the drive forms some $\mathrm{r} 2$ resistance alleles, they would show the same phenotype as drive alleles, thus only somewhat slowing the spread of the gene drive and likely still allowing successful suppression ${ }^{41}$. Functional r1 resistance alleles, on the other hand, would be expected to have a drastic effect on this type of drive, quickly halting and reversing population suppression and outcompeting the drive $\mathrm{e}^{26,34,42-44}$. Thus, the success of a suppression drive hinges on its ability to reduce the r1 allele 
formation rate to a sufficiently low level while also avoiding gRNA targets where r1 alleles are already present in the population.

The formation of such functional $\mathrm{r} 1$ resistance alleles was successfully prevented in one Anopheles study targeting a highly conserved sequence of a female fertility gene, since endjoining repair of such a target would be unlikely to result in a functional mutation ${ }^{29}$. However, the population size in this experimental study was necessarily limited to several hundred individuals, so it remains unclear if any functional $\mathrm{r} 1$ resistance alleles could form against this drive in much larger natural populations. It is therefore possible that additional measures may be needed in a large-scale release to prevent the formation of functional resistance alleles. Furthermore, such highly conserved sequences in possible target genes for suppression drives may not be available in other species.

Multiplexing gRNAs has been proposed as a mechanism that could reduce the rate of $\mathrm{r} 1$ allele formation by recruiting Cas9 to cleave at multiple sites within the target gene. If one gRNA target is repaired by end-joining in a way that leaves the gene functional, additional sites could still be cleaved, resulting in additional opportunities for drive conversion or creation of nonfunctional mutations. Simultaneous cleavage at multiple sites and repair by end-joining could also result in large deletions, which would usually render the target gene nonfunctional ${ }^{20}$. Several models indicate that multiplexed gRNAs would likely be effective at reducing functional resistance alleles ${ }^{24,45,46}$, and a handful of experimental studies have supported this notion ${ }^{11,20,24,25}$. Furthermore, multiplexing of gRNAs is capable of increasing drive conversion efficiency, as has been demonstrated in a modification homing drive with two gRNAs ${ }^{20}$. However, one study using four gRNAs for a homing suppression drive reported very low drive efficiency ${ }^{11}$, which would likely prevent effective population suppression ${ }^{24,44}$, particularly in larger, spatially-structured populations ${ }^{42,47}$. This reduction in efficiency was in part caused by repetitive elements in the drive $^{11}$, but widely spaced gRNAs also likely played a role ${ }^{24}$.

These findings suggest that an effective suppression drive could consist of multiple gRNAs targeting closely spaced sequences. The best target would likely be a female-specific haplosufficient but essential fertility gene ${ }^{42}$. Although such a drive would impose a high fitness cost to homozygous females, it could still spread at a high rate through germline conversion in heterozygous females and males, and any $\mathrm{r} 2$ resistance alleles would eventually be removed from the population rather than outcompeting the drive. Females with any combination of drive and $\mathrm{r} 2$ resistance alleles would be infertile.

Here, we construct such a drive in Drosophila melanogaster with four multiplexed gRNAs targeting yellow-g. The homing suppression drive demonstrated in these experiments showed elevated inheritance rates and successfully persisted in cage populations that averaged over 4,000 flies per generation without apparent formation of $\mathrm{r} 1$ resistance alleles. However, the drive also imposed an unintended fitness cost that ultimately prevented suppression of the experimental populations. 


\section{METHODS}

Plasmid construction. The starting plasmids TTTgRNAtRNAi ${ }^{24}$, TTTgRNAt $^{24}$, and BHDcN1 ${ }^{20}$ were constructed previously. For plasmid cloning, reagents for restriction digest, PCR, and Gibson assembly were obtained from New England Biolabs; oligos and gBlocks from Integrated DNA Technologies; 5- $\alpha$ competent Escherichia coli from New England Biolabs; and the ZymoPure Midiprep kit from Zymo Research. Plasmid construction was confirmed by Sanger sequencing. A list of DNA fragments, plasmids, primers, and restriction enzymes used for cloning of each construct can be found in the Supplemental Information section. We provide annotated sequences of the final drive insertion plasmid and target gene genomic region in ApE format at github.com/MesserLab/HomingSuppressionDrive (for the free ApE reader, see biologylabs.utah.edu/jorgensen/wayned/ape).

Generation of transgenic lines. Embryo injections were provided by Rainbow Transgenic Flies. The donor plasmid HSDygU4 was injected into $w^{1118}$ flies along with plasmid TTTygU4, providing the gRNAs for transformation, and pBS-Hsp70-Cas9 $(140 \mathrm{ng} / \mu \mathrm{L}$, from Melissa Harrison \& Kate O'Connor-Giles \& Jill Wildonger, Addgene plasmid \#45945) as the source of Cas9 for transformation.

Genotypes and phenotypes. Flies were anesthetized with $\mathrm{CO}_{2}$ and screened for fluorescence using the NIGHTSEA adapter SFA-GR for DsRed and SFA-RB-GO for EGFP. Fluorescent proteins were driven by the $3 \mathrm{xP} 3$ promoter for expression and easy visualization in the white eyes of $w^{1118}$ flies. DsRed was used as a marker to indicate the presence of a drive allele, and EGFP was used to indicate the presence of Cas9 $9^{22}$.

Fly rearing. Flies were housed with BDSC standard cornmeal medium in a $25^{\circ} \mathrm{C}$ incubator on a 14/10-hour day/night cycle. For the cage study, flies were housed in 30x30x30 cm (Bugdorm, BD43030D) enclosures. The ancestral founder line that was heterozygous for the drive allele and homozygous for the split-Cas 9 allele was generated by crossing successful transformants with the Cas9 line ${ }^{22}$ for several generations, selecting flies with brighter green fluorescence (which were likely to be Cas9 homozygotes) and eventually confirming that the line was homozygous for Cas9 via PCR.

These flies (heterozygous for the drive and homozygous for Cas9), together with split-Cas922 homozygotes of the same age, were separately allowed to lay eggs in eight food bottles for a single day. Bottles were then placed in cages, and eleven days later, they were replaced in the cage with fresh food. Bottles were removed from the cages the following day, the flies were frozen for later phenotyping, and the egg-containing bottles returned to the cage. This 12-day cycle was repeated for each generation. 
All gene drive flies were held at Cornell University's Sarkaria Arthropod Research Laboratory under Arthropod Containment Level 2 protocols with USDA APHIS standards. All safety measures were approved by the Cornell University Institutional Biosafety Committee.

Phenotype data analysis. Data were pooled into two groups of all female crosses or all male crosses in order to calculate drive inheritance, drive conversion, and embryo resistance. However, this pooling approach does not take potential batch effects (offspring were raised in different vials with different parents) into account, which could bias rate and error estimates. To account for such batch effects, we conducted an alternate analysis as in previous studies ${ }^{24,25,48}$. Briefly, we fit a generalized linear mixed-effects model with a binomial distribution (by maximum likelihood, Adaptive Gauss-Hermite Quadrature, $\mathrm{nAGQ}=25$ ). This model allows for variance between batches, usually resulting in slightly different parameter estimates and increased error estimates. Offspring from a single vial were considered as a separate batch. This analysis was performed with the $\mathrm{R}$ statistical computing environment (3.6.1) including packages lme4 (1.1-21, https://cran.r-project.org/web/packages/lme4/index.html) and emmeans (1.4.2, https://cran.r-project.org/web/packages/emmeans/index.html). The script is available on Github (https://github.com/MesserLab/Binomial-Analysis). The resulting rate estimates and errors were similar to the pooled analysis (Data Sets S1-3).

Genotyping. For genotyping, flies were frozen, and DNA was extracted by grinding flies in 30 $\mu \mathrm{L}$ of $10 \mathrm{mM}$ Tris-HCl pH 8, 1mM EDTA, $25 \mathrm{mM} \mathrm{NaCl}$, and $200 \mu \mathrm{g} / \mathrm{mL}$ recombinant proteinase $\mathrm{K}$ (ThermoScientific), followed by incubation at $37^{\circ} \mathrm{C}$ for 30 minutes and then $95^{\circ} \mathrm{C}$ for 5 minutes. The DNA was used as a template for PCR using Q5 Hot Start DNA Polymerase from New England Biolabs with the manufacturer's protocol. The region of interest containing gRNA target sites was amplified using DNA oligo primers YGLeft_S_F and YGRight_S_R. This would allow amplification of wild-type sequences and sequences with resistance alleles but would not amplify full drive alleles with a 30 second PCR extension time. After DNA fragments were isolated by gel electrophoresis, sequences were obtained by Sanger sequencing and analyzed with ApE software (http://biologylabs.utah.edu/jorgensen/wayned/ape).

Fitness cost inference framework. To quantify drive fitness costs, we modified a previously developed maximum likelihood inference framework ${ }^{25,49}$. Similar to a previous study ${ }^{50}$, we extended the model to two unlinked loci (drive site and an a site representing undesired mutations from off-target cleavage that impose a fitness cost). The Maximum Likelihood inference framework is implemented in R (v. 4.0.3) $)^{51}$ and is available on GitHub (github.com/MesserLab/HomingSuppressionDrive).

In this model, we make the simplifying assumption of a single genetic loci and a single gRNA at the gene drive allele site. Each female randomly selects a mate. The number of offspring generated per female can be reduced in certain genotypes if they have a fecundity fitness cost, and the chance of a male being selected as a mate can be reduced if they have a mating success fitness cost. In the germline, wild-type alleles in drive/wild-type heterozygotes can potentially be 
converted to either drive or resistance alleles, which are then inherited by offspring. At this stage, wild-type alleles at the off-target site are also cleaved, becoming disrupted alleles that may impose a fitness cost. The genotypes of offspring can be adjusted if they have a drive-carrying mother. If they have any wild-type alleles, then these are converted to resistance alleles at the embryo stage. This final genotype is used to determine if the offspring survives based on viability fitness.

We set the germline drive conversion rate and the embryo resistance allele formation rate to the experimental inferred estimates $(76.7 \% ; 52.2 \%)$. Based on previous observations ${ }^{19,20,22,24}$, we set the germline $\mathrm{r} 2$ formation rate to $22.2 \%$ so that nearly all wild-type alleles would either be converted to a drive allele or a resistance allele. $r 1$ alleles were not initially modeled since they are expected to be extremely rare in the 4-gRNA design (but see below). Note that in this framework, drive conversion and germline resistance allele formation take place at the same temporal stage in the germline. We set the germline cut rate at the off-target locus to 1 and did not model additional off-target cuts in embryos with drive-carrying mothers. We assumed that in drive carriers at the beginning of the experiment, $50 \%$ of the off-target sites are cut because the drive carrier flies all came from male drive heterozygotes. All drive carriers were initially drive heterozygotes. In future generations, we used the relative rate of drive heterozygotes and homozygotes (among drive carriers with DsRed) as well as relative rates of other genotypes with a wild-type (non-DsRed) phenotype as predicted in the maximum likelihood model.

In one model, we assumed the fitness costs would occur only in female drive/wild-type heterozygotes due to somatic Cas9 expression and cleavage. In the remaining scenarios, we assumed that drive fitness costs would either reduce viability or reduce female fecundity (separately from the sterility of female drive homozygotes) and male mating success. These fitness costs either stemmed directly from the presence of the drive or from cleavage at a single off-target site (representing multiple possible off-target sites that were unlinked to the drive). Our fitness parameters represent the fitness of drive homozygotes (or simply the net fitness of drive heterozygotes for the somatic Cas 9 cleavage fitness model). Heterozygous individuals were assigned a fitness equal to the square root of homozygotes, assuming multiplicative fitness costs between loci. The model incorporates the sterility of females not carrying any wild-type allele of yellow- $g$, and thus, any inferred fitness parameters $<1$ represent additional fitness costs of the drive system.

To estimate the rate at which resistance alleles might be functional r1 types, we took the best model for each cage and introduced a new "r1 rate" parameter, representing the fraction of resistance alleles that become $\mathrm{r} 1$ alleles instead of $\mathrm{r} 2$ alleles. Our germline rate of $22.2 \%$ then became the total resistance allele formation rate, while the experimental measured embryo $\mathrm{r} 2$ allele formation rate remained fixed at $52.2 \%$. This relative $\mathrm{r} 1$ rate parameter was then inferred as above to obtain an estimate and confidence interval. 


\section{RESULTS}

Drive construct design. In this study, we aim to develop a population suppression homing drive in D. melanogaster that utilizes multiple gRNAs to improve drive efficiency and reduce the rate of functional $\mathrm{r} 1$ resistance allele formation. Our drive construct targets yellow- $\mathrm{g}$, which is located on chromosome $3 \mathrm{~L}$ and has been shown to play a critical role in egg development ${ }^{52}$. Null mutations of yellow- $g$ usually result in sterile females when homozygous but show no effects on male fertility or on females when one wild-type copy is present (Figure 1). Both integration of the drive or formation of $\mathrm{r} 2$ resistance alleles that disrupt gene function will result in such null alleles. Conversion of wild-type alleles to drive alleles in the germline of drive heterozygotes allows the drive to increase in frequency in the population (Figure 1). This will lead to an increasing number of sterile individuals that can eventually induce population suppression.

A

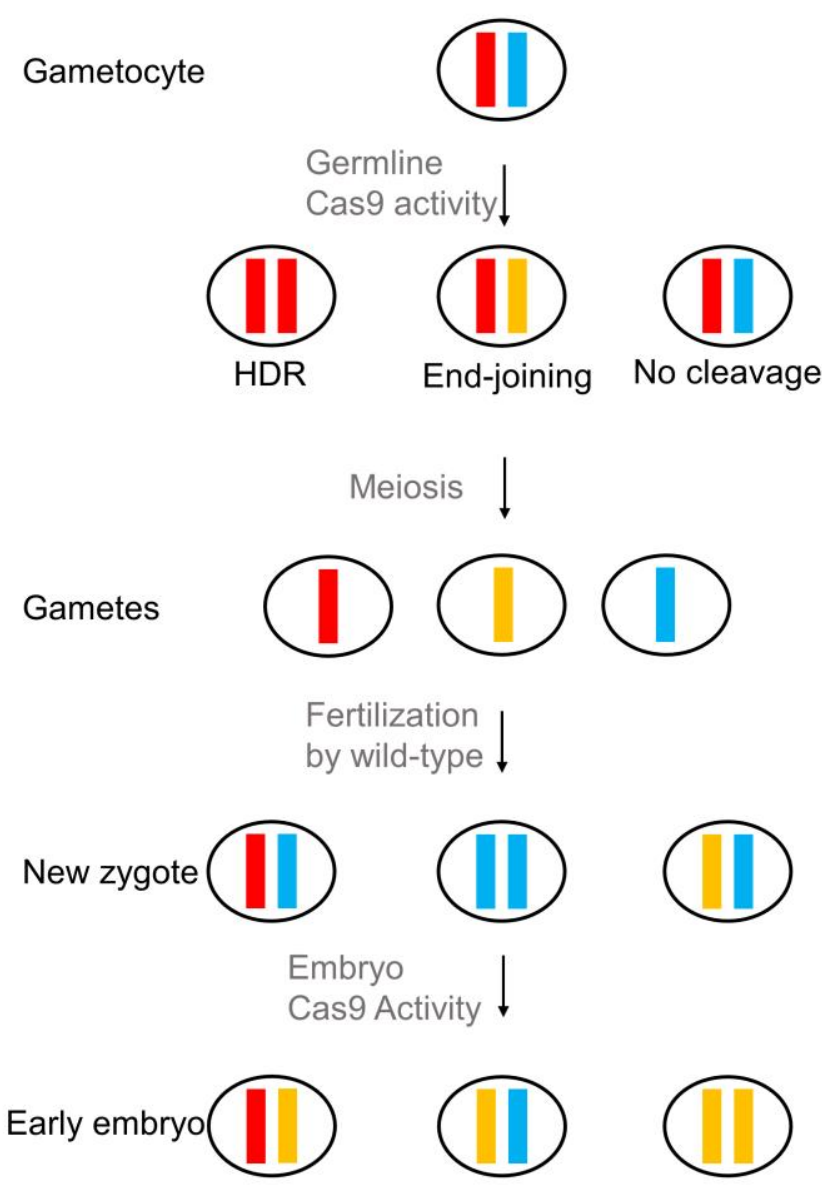

B Genotypes
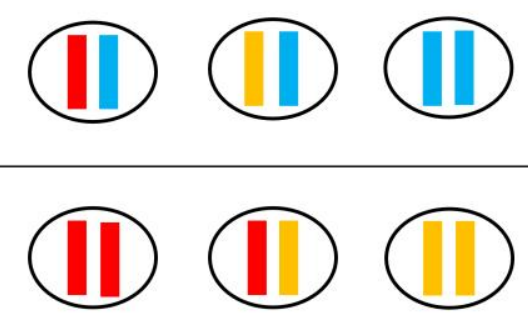

Female-sterile genotypes

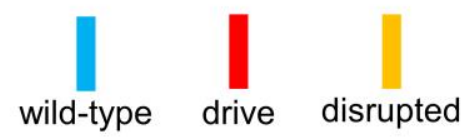

Figure 1. Homing suppression drive inheritance. (A) Germline Cas9 activity can convert wild-type allele to drive alleles, though end-joining repair can produce resistance alleles. Maternally deposited Cas9 and gRNA can form additional resistance alleles in the early embryo. (B) Females carrying only drive alleles or $\mathrm{r} 2$ resistance alleles are sterile.

The drive is inserted between the leftmost and rightmost gRNA target sites of yellow- $g$, providing the template for homology directed repair (Figure 2). The drive construct contains a 
DsRed fluorescent marker driven by the $3 \mathrm{xP} 3$ promoter for expression in the eyes to indicate the presence of a drive allele. It also contains four gRNAs (confirmed to be active by target sequencing) within tRNA scaffolding that target the second exon of yellow-g. By eliminating the need for multiple gRNA cassettes, the construct is more compact and avoids repetitive gRNA promoter elements. All four gRNA target sites are located within the second exon of yellow-g. This design should both increase the drive's homing rate as well as the probability that when a resistance allele is formed, it is an r2 allele (which disrupts the target gene's function) rather than an $\mathrm{r} 1$ allele (which preserves the function). The Cas9 element, required for drive activity, is placed on chromosome $2 \mathrm{R}$ and provided through a separate line that carries Cas 9 driven by the nanos germline promoter and EGFP with the $3 \mathrm{xP} 3$ promoter. In this split-Cas9 system, the drive will only be active in individuals where the Cas9 allele is also present ${ }^{22}$.

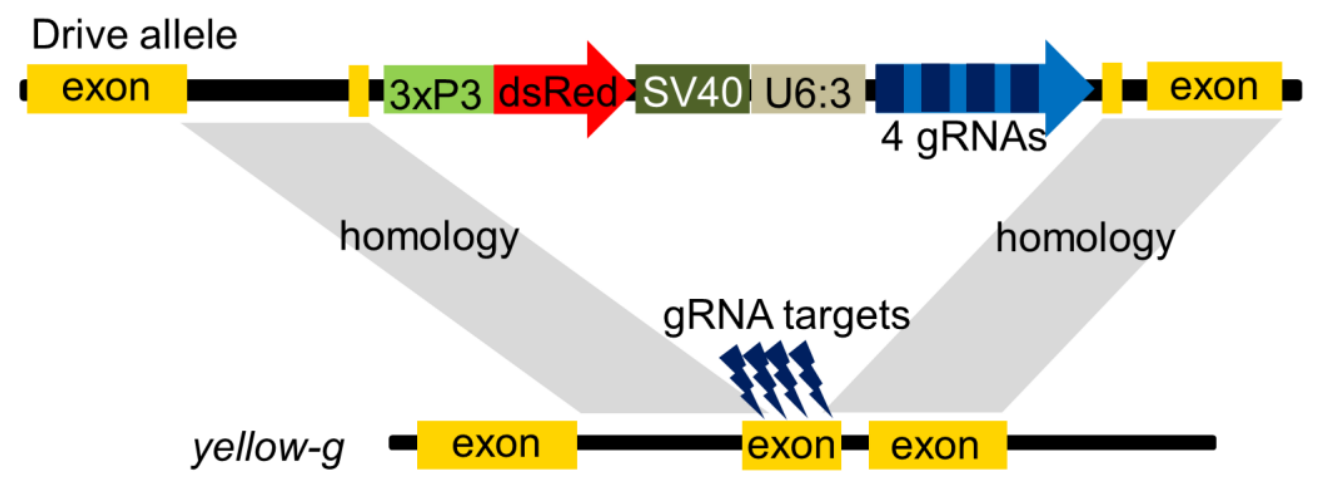

Figure 2. Homing suppression drive schematic. The drive is placed inside the yellow- $g$ gene at the gRNA target sites to allow for homology directed repair. A DsRed fluorescence marker is driven by the $3 \mathrm{xP} 3$ promoter. Four gRNAs (multiplexed in tRNA scaffolding and driven by the U6:3 promoter) target regions of the second exon of yellow-g. This is a split drive system, so Cas9 (driven by the nanos promoter) was provided at an unlinked site in the genome for drive experiments.

Drive inheritance. Successful transformants were used to establish fly lines with the construct, which were maintained by removing wild-type females in each generation. We first crossed the drive line to a line that was homozygous for nanos-Cas9. The offspring of this cross that had DsRed are expected to carry one copy each of the drive allele and the Cas9 allele, and these flies were then crossed with $w^{1118}$ flies for drive conversion assessment. The offspring of this second cross were phenotyped for red fluorescence, indicating the presence of the drive allele (Figure 3) and in a subset of the vials, also for green fluorescence, indicating presence of the Cas 9 allele. The drive was inherited at a rate of $86.4 \%$ in the progeny of female drive heterozygotes (Data Set $\mathrm{S} 1$ ), substantially higher than the Mendelian inheritance rate of 50\% (Fisher's Exact Test, $p<$ 0.00001 ) and thus indicative of strong drive activity. For the progeny of male drive heterozygotes, the inheritance rate was $90.4 \%$ (Data Set S2), which was also substantially higher than the Mendelian expectation (Fisher's Exact Test, $p<0.00001$ ). Because we do not expect this drive to reduce the viability of any eggs, we can calculate the rate at which wild-type alleles were converted to drive alleles based on the drive inheritance rate. This drive conversion rate was $72.7 \%$ for female heterozygotes and $80.7 \%$ for male heterozygotes. These rates were greater 
than most similar single gRNA designs ${ }^{19,20,22}$ and comparable to similar 2-gRNA designs ${ }^{20,24,25}$, as predicted by a model of multiple $\mathrm{gRNAs}^{24}$.

The inheritance rate of the Cas9 allele (which should have unbiased inheritance) was $46.7 \%$ for females and $43.8 \%$ for males. These rates were not significantly different from the Mendelian expectation of $50 \%$ ( $p=0.3$ for females and $p=0.1$ for males, Binomial test), consistent with little to no fitness costs for the Cas 9 cassette.

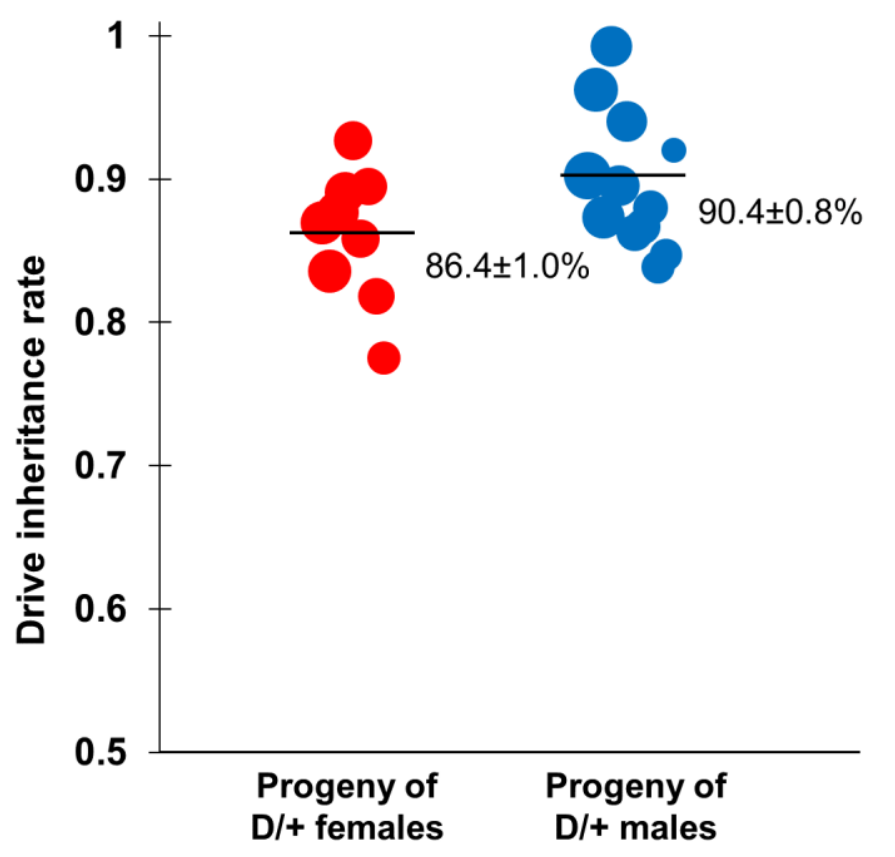

Figure 3. Drive inheritance rates. Drive inheritance as measured by the percentage of offspring with DsRed fluorescence from crosses between drive individuals (heterozygous for the drive and for a Cas9 allele) and wild-type flies. Each dot represents offspring from one drive parent, and the size of dots is proportional to the number of total offspring from the parent. Rate and standard error of the mean are displayed for the overall inheritance rate for all flies pooled together. An alternate analysis that accounts for potential batch effects yielded overall similar rates with slightly increased error estimates (Data Sets S1-2).

Embryo resistance and fertility. To determine the rate of resistance allele formation in the embryo due to maternally deposited Cas 9 and gRNAs, DsRed female offspring were assessed for fertility. These individuals were daughters of drive heterozygous mothers (heterozygous for both drive and Cas9 alleles and crossed to $w^{1118}$ males as described above) and could thus have developed embryo resistance. This would convert these flies from fertile drive/wild-type heterozygotes into drive/r2 resistance allele heterozygotes, which are expected to be sterile.

Twelve out of $22(54.5 \%)$ assessed females were sterile, which is significantly higher than the $5 \%$ sterility rate (in one out of 20 individuals tested) of female drive-carrier offspring from male drive and Cas9 heterozygotes crossed to $w^{1118}$ females ( $p<0.001$, Fisher's exact test). Assuming that this $5 \%$ sterility rate represents a baseline for our laboratory flies under the given experimental conditions, we can calculate that an embryo resistance allele formation rate of 
$52.2 \%$ will account for the increased sterility rate in the progeny of drive females. This should provide an estimate for the rate at which the paternal wild-type alleles of yellow-g were cleaved at one or more gRNA target sites in embryos with drive mothers.

Fertility and viability. Homozygous females (as confirmed by sequencing) were found to be sterile as expected. One important issue with population suppression gene drives is leaky somatic expression that can convert drive/wild-type heterozygotes partially or completely into drive/resistance allele heterozygotes (or perhaps even drive/drive homozygotes) in somatic cells, which was responsible for substantially reducing the fertility of mosquitoes carrying homing suppression drives in previous studies ${ }^{26,29,34}$. To determine if drive heterozygotes had altered fertility, three-day old female virgins that were heterozygous for the drive and Cas 9 alleles were crossed with $w^{1118}$ males and then allowed to lay eggs for three consecutive days in different vials, with the eggs counted each day. They laid an average \pm standard deviation of $33 \pm 4$ eggs per day (Data Set S1), which was significantly higher than the $20 \pm 2$ eggs per day laid by $w^{1118}$ females crossed to drive and Cas9 heterozygous males (Data Set S2, $p=0.008$, t-test) or the $23 \pm 2$ eggs per day laid by $w^{1118}$ females crossed with $w^{1118}$ males (Data Set S3, $p=0.017$, t-test). This greater number of eggs per day was likely a batch effect from perhaps slightly older or healthier drive females compared to the $w^{1118}$ females used. Indeed, if the first day of egg-laying is discounted, the new average of $25 \pm 3$ eggs per day for drive heterozygous females is statistically indistinguishable from the other groups, regardless of whether the first day of egglaying is retained in these groups ( $p>0.1$ for all comparisons, t-test). This indicates that any drive cleavage from leaky somatic expression is sufficiently low, such that it does not substantially reduce female fertility (though we cannot rule out small reductions). These results are consistent with the notion that the nanos-Cas9 allele has little to no leaky somatic expression, as shown in previous Drosophila studies ${ }^{19,20,22,25}$.

The offspring of these crosses (females heterozygous for the drive and Cas 9 crossed with $w^{1118}$ males, males heterozygous for the drive and Cas9 crossed with $w^{1118}$ females, and $w^{1118}$ fly crosses) did not exhibit any apparent developmental fitness costs. In particular, there were no differences in egg or pupae viability between these three groups of offspring (Data Sets S1-S3).

Cage study. To assess the ability of our homing suppression drive to spread over the course of several generations, we conducted a cage study. Flies heterozygous for the drive and homozygous for Cas9 were introduced into two cages at frequencies of $41 \%$ and $8.8 \%$ and were allowed to lay eggs in bottles for one day. Flies homozygous for the Cas 9 allele were similarly allowed to lay eggs in separate bottles. We then removed the flies and placed the bottles together in each population cage. The cages were followed for several generations, with each generation phenotyped for DsRed to measure the drive carrier frequency, which includes drive homozygotes and heterozygotes. In both cages, the drive carrier frequency increased to approximately $63 \%$ (Figure 4, Data Set S4). This possibly represents an equilibrium frequency, though the cages would perhaps have increased to a somewhat higher equilibrium frequency with additional generations (models of this drive type predict an asymptotic approach to the equilibrium 
frequency ${ }^{24}$, making it difficult to estimate from limited cage data). However, either of these values is substantially lower than the expected drive carrier equilibrium frequency of approximately $90 \%$, given the average drive conversion rate in heterozygotes of $76.7 \%{ }^{24,41,44}$ (for a simple models of homing suppression drives with one gRNA - a more advanced model would predict a marginally higher equilibrium frequency ${ }^{24}$ ). In these models, such a reduction in equilibrium frequency could perhaps be explained by a fitness cost of $15-20 \%$ in drive homozygotes (with multiplicative fitness costs) ${ }^{24}$. Nevertheless, the drive frequency did not decrease systematically over the course of the experiment after the initial increase of the drive, suggesting that functional $\mathrm{r} 1$ resistance alleles did not form at a high rate. This is in contrast to two recent studies of homing suppression drives where $\mathrm{r} 1$ alleles outcompeted the drive alleles despite lower population sizes and higher drive efficiency ${ }^{26,34}$.

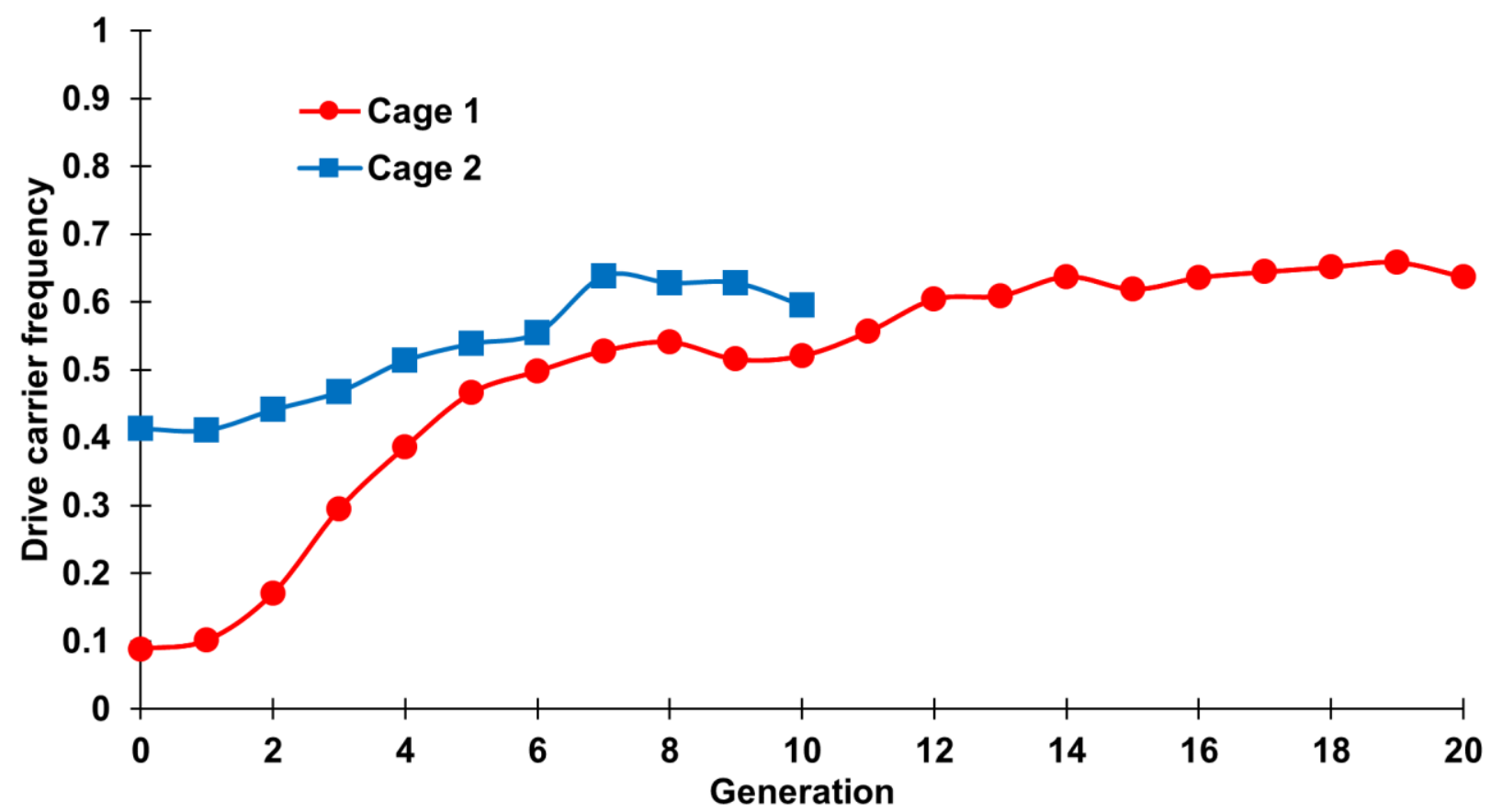

Figure 4. Frequency of drive carriers in cage. Flies carrying one copy of the drive allele and two copies of Cas 9 were introduced at initial frequencies of $8.8 \%$ (cage 1) and $41.3 \%$ (cage 2) into a population that was wild-type at the drive site and homozygous for the Cas9 allele. The cage populations were followed for several non-overlapping generations, each lasting twelve days, including one day of egg-laying. All individuals from each generation were phenotyped for DsRed, with positive drive carriers having either one or two drive alleles (all drive carriers in the initial generation were drive/wild-type heterozygotes).

A substantial reduction in the population size for this homing suppression drive was not observed (Figure S1). This is likely due to the modest genetic load of the drive, which we use as a measure of the reduction of reproductive capacity of a population $(0=$ no loss of reproductive capacity, 1 $=$ the population can no longer reproduce). The load of our drive is closely related to the proportion of sterile females in the population, which increases with drive frequency. In our cages, drive frequency only reached a moderate level, thus imposing only a moderate genetic load. This genetic load was lower than expected because the drive appeared to carry a fitness 
cost, which would directly reduce the drive's equilibrium frequency and genetic load. Additionally, with the flies likely laying an average of over 20 eggs per female (Data Sets S1-S3) and with reduced larval densities usually leading to healthier adults (which could perhaps mature faster and lay even more eggs due to greater size obtained as larva), this would require a particularly high genetic load to reduce the population at all. While the drive may still have caused a small population reduction, this was not detectable given the level of population size fluctuation between generations.

To further investigate the nature of the drive's fitness costs, a new cage was established with flies both homozygous and heterozygous for the drive allele but lacking the Cas 9 allele required for homing. Such flies were placed in a cage at an initial drive carrier frequency of $76 \%$. Over ten generations, the drive-carrier frequency declined to 29\% (Figure S2). This observation is consistent with the drive allele being a recessive female sterile allele (as expected from its disruption of yellow-g) and having no additional fitness costs in the absence of a genomic source of Cas9. The previous experiments indicated low somatic expression and similar fecundity of drive flies compared to $w^{1118}$ flies. Thus, fitness costs are apparently due to the drive allele itself in conjunction with Cas9, or at least individual fitness components are sufficiently small that these experiments did not reveal differences between drive and wild-type.

Maximum likelihood analysis of cage data. To computationally assess drive performance, we adapted a previously developed method ${ }^{25,49}$ to infer fitness costs based on phenotype data from population cages. We used a simplified model that included only a single gRNA and initially neglected possible formation of functional resistance alleles, assuming that all resistance alleles were nonfunctional. Note that this simplifying assumption of one gRNA for a drive with four slightly underestimates drive performance compared to a more complex model ${ }^{24}$ with the same parameters for drive-wild-type heterozygotes. Since drive carrier individuals in the initial generation of all three cages clearly had substantially lower fitness than in other generations (most likely due differences in health in the populations of the initial generation), likelihood values for the transition from the initial generation to the next were excluded from the analysis.

We reason that the first cage is more reliable for parameter estimation due to the greater number of generations and lower starting frequency, allowing more generations in which the drive can increase toward its possible equilibrium frequency. This equilibrium is predicted by models of homing suppression drives that match the design of our drive ${ }^{24,41,44}$. In this cage, a model of viability-based fitness costs had the best fit to the data based on the Akaike information criterion corrected for small sample size, with drive homozygotes having a viability of $80 \%(95 \%$ confidence interval: 72-88\%) compared to wild-type individuals (Table S1A). We did not observe reduced viability in our assays based on individual crosses, but these had limited power to detect such reduction in drive heterozygotes. Moreover, individually assayed flies probably did not experience the same intense competition that might be found in the cage populations. A model that included reduction of female fecundity and male mating success matched the data nearly as well as the viability model. 
In the second cage, a model with fitness costs from somatic Cas9 cleavage of yellow- $g$ in female drive/wild-type heterozygotes was the best match to the data (Table S1B), with such females having a $57 \%$ reduction in fecundity. However, this result is not consistent with our direct measurements of fecundity for drive heterozygous females (Data Sets S1-S3). A model with offtarget viability fitness costs due to Cas9 cleavage of distant sites was the next best match, though this model did not perform well in the first cage.

We did not observe qualitative evidence of functional $\mathrm{r} 1$ alleles in our cages, which would have resulted in a systematic decline in drive frequency toward the end of the experiments when present at a high enough rate. Furthermore, our maximum likelihood method allows us to estimate an upper bound of the maximum rate at which $\mathrm{r} 1$ alleles may have been formed in our population cages. To accomplish this, we used the best fitting model above for each of the two experimental cages and allowed the $\mathrm{r} 1$ rate to vary, assuming it to be proportional to the nonfunctional $\mathrm{r} 2$ resistance allele formation rate. In both cages, the most likely estimate was $0 \%$. The first cage had an $95 \%$ confidence upper bound of $0.225 \%$ for the relative $\mathrm{r} 1$ formation rate (fraction of total resistance alleles that were $\mathrm{r} 1$ alleles), which corresponds to germline and embryo $\mathrm{r} 1$ resistance allele formation rates of $0.050 \%$ and $0.12 \%$, respectively. The second cage, with its smaller number of generations, lacked the power to provide a tightly bounded relative r1 rate estimate, with an upper $95 \%$ confidence level of $19 \%$. Note that computational models predict even lower rates of functional resistance allele formation in 4-gRNA drives, likely below $0.01 \%$ the level of the $\mathrm{r} 2$ rate, perhaps even lower by several orders of magnitude if the rate of functional sequence repair at individual sites is well below $10 \%{ }^{24}$.

In the control cage lacking Cas9, the drive declined as expected for an allele that caused recessive sterility in females, with no additional fitness costs (Table S1C). This indicates that any fitness costs are likely due to the drive itself together with Cas9, rather than haploinsufficiency of yellow- $g$ or an effect in males.

\section{DISCUSSION}

This study experimentally demonstrated the utility of gRNA multiplexing as a means for improving the ability of a homing suppression drive to spread through a population without significant formation of functional $\mathrm{r} 1$ resistance alleles. The drive displayed a higher drive conversion rate than most single gRNA Drosophila drive systems ${ }^{19,20,22}$, as well as a previous homing suppression drive with four gRNAs ${ }^{11}$. However, it had a moderate embryo resistance rate that presumably reduced its rate of spread through the cage. The drive also carried a small fitness cost of unknown origin, which reduced the genetic load of the drive on the population, ultimately preventing suppression. Notwithstanding, this study still demonstrated an additional strategy against $\mathrm{r} 1$ resistance allele formation in suppression drives that is complementary to the targeting of highly conserved sites, which was previously demonstrated as an effective 
approach $^{29}$. Combined, these strategies would likely be even more powerful at reducing the formation of $\mathrm{r} 1$ resistance alleles while still maintaining high drive conversion efficiency ${ }^{24}$.

Since genetic load (which determines the suppressive power of a drive) is mostly determined by drive conversion efficiency and fitness costs, this represents a hurdle for any suppression strategy based on a homing drive. As the frequency of the drive increases, so does the rate of drive removal. With $100 \%$ homing efficiency, the relative frequency of the drive allele would continue to increase as the population numbers decline, and complete suppression would occur as the drive reaches fixation. However, with a lower efficiency, wild-type alleles remain, and the antagonistically acting forces of drive conversion and drive allele removal result in an equilibrium frequency. Fitness costs from the drive would, in this case, further reduce the equilibrium drive frequency and resulting genetic load. Homing suppression drives in mosquitoes $^{27,29,34}$, therefore, have demonstrated superior genetic loads compared to this drive in fruit flies. In these mosquito drives, the higher somatic fitness costs were more than compensated for by a higher drive conversion efficiency. Engineering sufficiently high drive conversion efficiency could therefore be a challenge when designing drives for suppression in Drosophilids such as Drosophila suzukii. Additionally, the reduced equilibrium represents a limitation in our study, since at a lower equilibrium frequency, functional $\mathrm{r} 1$ resistance alleles have a reduced fitness advantage compared to drive and wild-type alleles, making them more difficult to detect using our maximum likelihood method that analyzes drive carrier frequency trajectories. Our analysis of $\mathrm{r} 1$ alleles also depended on the fit of our model with drive efficiency and fitness parameters, with the latter in particular being difficult to accurately assess.

In fact, it remains unclear exactly what caused the fitness costs associated with our homing suppression drive. Though we could estimate the magnitude of such costs, their nature could not be determined based on our data. It is possible that a combination of several different types of fitness costs was at play, including fitness components that we did not include in our model. For example, perhaps the target gene was slightly haploinsufficient, which could potentially be addressed by changing the target gene to one of many other possible female fertility genes. Another possibility is off-target cleavage effects as seen previously ${ }^{50}$, which would likely be exacerbated by multiplexed gRNAs. However, such an issue could be addressed relatively easily by using high fidelity Cas9 nucleases that show little to no off-target cleavage ${ }^{53-59}$, which have been shown to have similar drive performance ${ }^{50}$. By contrast, if fitness costs are caused by the expression of the drive components themselves, they may be more difficult to directly address. In this case, increasing drive conversion efficiency, for example by using a different Cas9 promoter, may be the best route to developing successful drives. Indeed, modeling indicates that high drive efficiency and fitness may play an even more important role in ensuring success in complex natural populations with spatial structure ${ }^{42,47,60-62}$.

If the drive conversion rate is insufficient for a given set of available genetic tools, then a TADE suppression drive ${ }^{63,64}$ may still be able to provide a high genetic load since it only requires high efficiency for germline cleavage (regardless of whether it results in homology-directed repair or 
end-joining) rather than for the drive conversion process (which requires homology-directed repair). Though engineering such a drive targeting haplolethal genes may be challenging, working with such genes is possible at least for homing drives ${ }^{25}$. However, TADE drives are frequency-dependent and thus weaker than homing drives, requiring higher release sizes for success ${ }^{63,64}$. In some cases, this feature may be desirable if the drive should be strictly confined to a target population. Another way to achieve confinement that could still involve a homing suppression drive would be to use a tethered system in which the split homing element is linked to a confined modification drive system ${ }^{65,66}$.

Overall, we have demonstrated that gRNA multiplexing can reduce functional $r 1$ resistance alleles in a homing suppression drive while maintaining a high drive conversion efficiency. Since multiplexing gRNAs is a fairly straightforward process, we believe this approach has the potential to be applied to a wide variety of suppression gene drive designs, providing similar benefits in many species.

\section{ACKNOWLEDGEMENTS}

This study was supported by the National Institutes of Health awards R21AI130635 to JC, AGC, and PWM, award F32AI138476 to JC, and award R01GM127418 to PWM.

\section{REFERENCES}

1. Champer J, Buchman A, Akbari OS. Cheating evolution: engineering gene drives to manipulate the fate of wild populations. Nat Rev Genet, 17, 146-159, 2016.

2. Burt A. Heritable strategies for controlling insect vectors of disease. Philos Trans $R$ Soc $L$ B Biol Sci, 369, 20130432, 2014.

3. Alphey L. Genetic control of mosquitoes. Annu Rev Entomol, 59, 205-224, 2014.

4. Esvelt KM, Smidler AL, Catteruccia F, Church GM. Concerning RNA-guided gene drives for the alteration of wild populations. Elife, e03401, 2014.

5. Hay BA, Oberhofer G, Guo M. Engineering the Composition and Fate of Wild Populations with Gene Drive. Annu Rev Entomol, 66, annurev-ento-020117-043154, 2021.

6. Burt A, Crisanti A. Gene Drive: Evolved and Synthetic. ACS Chem Biol, 13, 343-346, 2018.

7. DiCarlo JE, Chavez A, Dietz SL, Esvelt KM, Church GM. Safeguarding CRISPR-Cas9 gene drives in yeast. Nat Biotechnol, 33, 1250-1255, 2015.

8. Roggenkamp E, Giersch RM, Schrock MN, Turnquist E, Halloran M, Finnigan GC. Tuning CRISPR-Cas9 gene drives in Saccharomyces cerevisiae. G3, 8, 999-1018, 2018.

9. Basgall EM, Goetting SC, Goeckel ME, Giersch RM, Roggenkamp E, Schrock MN, Halloran M, Finnigan GC. Gene drive inhibition by the anti-CRISPR proteins AcrIIA2 and AcrIIA4 in Saccharomyces cerevisiae. Microbiology, 164, 464-474, 2018.

10. Shapiro RS, Chavez A, Porter CBM, Hamblin M, Kaas CS, DiCarlo JE, Zeng G, Xu X, Revtovich A V., Kirienko N V., Wang Y, Church GM, Collins JJ. A CRISPR-Cas9-based 
gene drive platform for genetic interaction analysis in Candida albicans. Nat Microbiol, 3 , 73-82, 2018.

11. Oberhofer G, Ivy T, Hay BA. Behavior of homing endonuclease gene drives targeting genes required for viability or female fertility with multiplexed guide RNAs. Proc Natl Acad Sci, 115, E9343-E9352, 2018.

12. KaramiNejadRanjbar M, Eckermann KN, Ahmed HMM, Sánchez C. HM, Dippel S, Marshall JM, Wimmer EA. Consequences of resistance evolution in a Cas9-based sexconversion suppression gene drive for insect pest management. Proc Natl Acad Sci, $201713825,2018$.

13. Chae D, Lee J, Lee N, Park K, Moon SJ, Kim HH. Chemical controllable gene drive in Drosophila. ACS Synth Biol, 2020.

14. López Del Amo V, Leger BS, Cox KJ, Gill S, Bishop AL, Scanlon GD, Walker JA, Gantz VM, Choudhary A. Small-Molecule Control of Super-Mendelian Inheritance in Gene Drives. Cell Rep, 31, 107841, 2020.

15. Kandul NP, Liu J, Buchman A, Gantz VM, Bier E, Akbari OS. Assessment of a split homing based gene drive for efficient knockout of multiple genes. G3 Genes, Genomes, Genet, 10, 827-837, 2020.

16. Xu X-RS, Bulger EA, Gantz VM, Klanseck C, Heimler SR, Auradkar A, Bennett JB, Miller LA, Leahy S, Juste SS, Buchman A, Akbari OS, Marshall JM, Bier E. Active Genetic Neutralizing Elements for Halting or Deleting Gene Drives. Mol Cell, 2020.

17. López Del Amo V, Bishop AL, Sánchez C HM, Bennett JB, Feng X, Marshall JM, Bier E, Gantz VM. A transcomplementing gene drive provides a flexible platform for laboratory investigation and potential field deployment. Nat Commun, 11, 1-12, 2020.

18. Gantz VM, Bier E. Genome editing. The mutagenic chain reaction: a method for converting heterozygous to homozygous mutations. Science (80- ), 348, 442-444, 2015.

19. Champer J, Reeves R, Oh SY, Liu C, Liu J, Clark AG, Messer PW. Novel CRISPR/Cas9 gene drive constructs reveal insights into mechanisms of resistance allele formation and drive efficiency in genetically diverse populations. PLoS Genet, 13, e1006796, 2017.

20. Champer J, Liu J, Oh SY, Reeves R, Luthra A, Oakes N, Clark AG, Messer PW. Reducing resistance allele formation in CRISPR gene drive. Proc Natl Acad Sci, 115, 5522-5527, 2018.

21. Champer J, Wen Z, Luthra A, Reeves R, Chung J, Liu C, Lee YL, Liu J, Yang E, Messer PW, Clark AG. CRISPR gene drive efficiency and resistance rate is highly heritable with no common genetic loci of large effect. Genetics, 2019.

22. Champer J, Chung J, Lee YL, Liu C, Yang E, Wen Z, Clark AG, Messer PW. Molecular safeguarding of CRISPR gene drive experiments. Elife, 8, 2019.

23. Guichard A, Haque T, Bobik M, Xu X-RS, Klanseck C, Kushwah RBS, Berni M, Kaduskar B, Gantz VM, Bier E. Efficient allelic-drive in Drosophila. Nat Commun, 10, 1640, 2019.

24. Champer SE, Oh SY, Liu C, Wen Z, Clark AG, Messer PW, Champer J. Computational and experimental performance of CRISPR homing gene drive strategies with multiplexed gRNAs. Sci Adv, 6, eaaz0525, 2020.

25. Champer J, Yang E, Lee E, Liu J, Clark AG, Messer PW. A CRISPR homing gene drive targeting a haplolethal gene removes resistance alleles and successfully spreads through a cage population. Proc Natl Acad Sci, 2020.

26. Hammond AM, Kyrou K, Bruttini M, North A, Galizi R, Karlsson X, Kranjc N, Carpi 
FM, D’Aurizio R, Crisanti A, Nolan T. The creation and selection of mutations resistant to a gene drive over multiple generations in the malaria mosquito. PLOS Genet, 13, e1007039, 2017.

27. Hammond A, Galizi R, Kyrou K, Simoni A, Siniscalchi C, Katsanos D, Gribble M, Baker D, Marois E, Russell S, Burt A, Windbichler N, Crisanti A, Nolan T. A CRISPR-Cas9 gene drive system targeting female reproduction in the malaria mosquito vector Anopheles gambiae. Nat Biotechnol, 34, 78-83, 2015.

28. Gantz VM, Jasinskiene N, Tatarenkova O, Fazekas A, Macias VM, Bier E, James AA. Highly efficient Cas9-mediated gene drive for population modification of the malaria vector mosquito Anopheles stephensi. Proc Natl Acad Sci U S A, 112, E6736-E6743, 2015.

29. Kyrou K, Hammond AM, Galizi R, Kranjc N, Burt A, Beaghton AK, Nolan T, Crisanti A. A CRISPR-Cas9 gene drive targeting doublesex causes complete population suppression in caged Anopheles gambiae mosquitoes. Nat Biotechnol, 2018.

30. Pham TB, Phong CH, Bennett JB, Hwang K, Jasinskiene N, Parker K, Stillinger D, Marshall JM, Carballar-Lejarazú R, James AA. Experimental population modification of the malaria vector mosquito, Anopheles stephensi. PLOS Genet, 15, e1008440, 2019.

31. Li M, Yang T, Kandul NP, Bui M, Gamez S, Raban R, Bennett J, Sánchez C HM, Lanzaro GC, Schmidt H, Lee Y, Marshall JM, Akbari OS. Development of a confinable gene drive system in the human disease vector aedes aegypti. Elife, 9, 2020.

32. Simoni A, Hammond AM, Beaghton AK, Galizi R, Taxiarchi C, Kyrou K, Meacci D, Gribble M, Morselli G, Burt A, Nolan T, Crisanti A. A male-biased sex-distorter gene drive for the human malaria vector Anopheles gambiae. Nat Biotechnol, 1-7, 2020.

33. Carballar-Lejarazú R, Ogaugwu C, Tushar T, Kelsey A, Pham TB, Murphy J, Schmidt H, Lee Y, Lanzaro GC, James AA. Next-generation gene drive for population modification of the malaria vector mosquito, Anopheles gambiae. Proc Natl Acad Sci U S A, 117, 22805-22814, 2020.

34. Hammond A, Karlsson X, Morianou I, Kyrou K, Beaghton A, Gribble M, Kranjc N, Galizi R, Burt A, Crisanti A, Nolan T. Regulating the expression of gene drives is key to increasing their invasive potential and the mitigation of resistance. PLOS Genet, 17, e1009321, 2021.

35. Adolfi A, Gantz VM, Jasinskiene N, Lee HF, Hwang K, Terradas G, Bulger EA, Ramaiah A, Bennett JB, Emerson JJ, Marshall JM, Bier E, James AA. Efficient population modification gene-drive rescue system in the malaria mosquito Anopheles stephensi. Nat Commun, 11, 1-13, 2020.

36. Grunwald HA, Gantz VM, Poplawski G, Xu X-RS, Bier E, Cooper KL. Super-Mendelian inheritance mediated by CRISPR-Cas9 in the female mouse germline. Nature, 566, 105109, 2019.

37. Unckless RL, Clark AG, Messer PW. Evolution of resistance against CRISPR/Cas9 gene drive. Genetics, 205, 827-841, 2017.

38. Noble C, Olejarz J, Esvelt K, Church G, Nowak M. Evolutionary dynamics of CRISPR gene drives. Sci Adv, 3, e1601964, 2017.

39. Li J, Aidlin Harari O, Doss A, Walling LL, Atkinson PW, Morin S, Tabashnik BE. Can CRISPR gene drive work in pest and beneficial haplodiploid species? Evol Appl, eva.13032, 2020.

40. Burt A. Site-specific selfish genes as tools for the control and genetic engineering of 
natural populations. Proc Biol Sci, 270, 921-928, 2003.

41. Beaghton AK, Hammond A, Nolan T, Crisanti A, Burt A. Gene drive for population genetic control: non-functional resistance and parental effects. Proceedings Biol Sci, 286, 20191586, 2019.

42. Champer J, Kim IK, Champer SE, Clark AG, Messer PW. Suppression gene drive in continuous space can result in unstable persistence of both drive and wild-type alleles. Mol Ecol, 30, 1086-1101, 2021.

43. Eckhoff PA, Wenger EA, Godfray HC, Burt A. Impact of mosquito gene drive on malaria elimination in a computational model with explicit spatial and temporal dynamics. Proc Natl Acad Sci U S A, 114, E255-e264, 2017.

44. Deredec A, Godfray HC, Burt A. Requirements for effective malaria control with homing endonuclease genes. Proc Natl Acad Sci U S A, 108, E874-80, 2011.

45. Prowse TAA, Cassey P, Ross J V, Pfitzner C, Wittmann TA, Thomas P. Dodging silver bullets: good CRISPR gene-drive design is critical for eradicating exotic vertebrates. Proceedings Biol Sci, 284, 20170799, 2017.

46. Marshall JM, Buchman A, Sanchez CH, Akbari OS. Overcoming evolved resistance to population-suppressing homing-based gene drives. Sci Rep, 7, 3776, 2017.

47. North AR, Burt A, Godfray HCJ. Modelling the suppression of a malaria vector using a CRISPR-Cas9 gene drive to reduce female fertility. BMC Biol, 18, 98, 2020.

48. Champer J, Lee E, Yang E, Liu C, Clark AG, Messer PW. A toxin-antidote CRISPR gene drive system for regional population modification. Nat Commun, 11, 1082, 2020.

49. Liu J, Champer J, Langmüller AM, Liu C, Chung J, Reeves R, Luthra A, Lee YL, Vaughn AH, Clark AG, Messer PW. Maximum likelihood estimation of fitness components in experimental evolution. Genetics, 211, 1005-1017, 2019.

50. Langmüller AM, Champer J, Lapinska S, Xie L, Liu J, Xu Y, Clark AG, Messer PW. Fitness effects of CRISPR endonucleases in Drosophila melanogaster populations. bioRxiv, 2021.

51. R Core Team. R: A language and environment for statistical computing. www.Rproject.org. 2018.

52. Claycomb JM, Benasutti M, Bosco G, Fenger DD, Orr-Weaver TL. Gene amplification as a developmental strategy: isolation of two developmental amplicons in Drosophila. Dev Cell, 6, 145-155, 2004.

53. Casini A, Olivieri M, Petris G, Montagna C, Reginato G, Maule G, Lorenzin F, Prandi D, Romanel A, Demichelis F, Inga A, Cereseto A. A highly specific SpCas9 variant is identified by in vivo screening in yeast. Nat Biotechnol, 36, 265-271, 2018.

54. Tan Y, Chu AHY, Bao S, Hoang DA, Kebede FT, Xiong W, Ji M, Shi J, Zheng Z. Rationally engineered Staphylococcus aureus Cas9 nucleases with high genome-wide specificity. Proc Natl Acad Sci U S A, 116, 20969-20976, 2019.

55. Chatterjee P, Jakimo N, Lee J, Amrani N, Rodríguez T, Koseki SRT, Tysinger E, Qing R, Hao S, Sontheimer EJ, Jacobson J. An engineered ScCas9 with broad PAM range and high specificity and activity. Nat Biotechnol, 1-5, 2020.

56. Slaymaker IM, Gao L, Zetsche B, Scott DA, Yan WX, Zhang F. Rationally engineered Cas9 nucleases with improved specificity. Science (80- ), 351, 84-88, 2016.

57. Lee J, Jung MH, Jeong E, Lee JK. Using Sniper-Cas9 to Minimize Off-target Effects of CRISPR-Cas9 Without the Loss of On-target Activity Via Directed Evolution. $J$ Vis Exp, $1-8,2019$. 
58. Xie H, Ge X, Yang F, Wang B, Li S, Duan J, Lv X, Cheng C, Song Z, Liu C, Zhao J, Zhang Y, Wu J, Gao C, Zhang J, Gu F. High-fidelity SaCas9 identified by directional screening in human cells. PLoS Biol, 18, e3000747, 2020.

59. Kleinstiver BP, Pattanayak V, Prew MS, Tsai SQ, Nguyen NT, Zheng Z, Joung JK. Highfidelity CRISPR-Cas9 nucleases with no detectable genome-wide off-target effects. Nature, 529, 490-495, 2016.

60. Dhole S, Lloyd AL, Gould F. Gene Drive Dynamics in Natural Populations: The Importance of Density Dependence, Space, and Sex. Annu Rev Ecol Evol Syst, 51, 505531, 2020.

61. Rode NO, Estoup A, Bourguet D, Courtier-Orgogozo V, Débarre F. Population management using gene drive: molecular design, models of spread dynamics and assessment of ecological risks. Conserv. Genet. 20, 671-690, 2019.

62. Bull JJ, Remien CH, Krone SM. Gene-drive-mediated extinction is thwarted by population structure and evolution of sib mating. Evol Med public Heal, 2019, 66-81, 2019.

63. Champer J, Kim IK, Champer SE, Clark AG, Messer PW. Performance analysis of novel toxin-antidote CRISPR gene drive systems. BMC Biol, 18, 27, 2020.

64. Champer J, Champer SE, Kim IK, Clark AG, Messer PW. Design and analysis of CRISPR-based underdominance toxin-antidote gene drives. Evol Appl, eva.13180, 2020.

65. Dhole S, Lloyd AL, Gould F. Tethered homing gene drives: a new design for spatially restricted population replacement and suppression. Evol Appl, eva.12827, 2019.

66. Metzloff M, Wang E, Dhole S, Clark AG, Messer PW, Champer J. Experimental demonstration of tethered gene drive systems for confined population modification or suppression. bioRxiv, 2021. 


\section{SUPPLEMENTARY INFORMATION}

\section{Supplementary Methods}

\section{Plasmid Construction:}

gRNA-tRNA Array:

\begin{tabular}{|l|l|l|l|}
\hline TTTygU4 & Template & Oligo/Enzyme 1 & Oligo/Enzyme 2 \\
\hline PCR Product & TTTgRNAtRNAi & YGg_g41_F & YGg_g41_R \\
\hline PCR Product & TTTgRNAt & YGg_g12_F & YGg_g12_R \\
\hline PCR Product & TTTgRNAt & YGg_g23_F & YGg_g23_R \\
\hline PCR Product & TTTgRNAt & YGg_g34_F & YGg_g34_R \\
\hline
\end{tabular}

Left Homology Arm:

\begin{tabular}{|l|l|l|l|l|}
\hline HSDygU4i1 & Template & Oligo/Enzyme 1 & Oligo/Enzyme 2 & Enzyme 3 \\
\hline PCR Product & Genomic DNA & YGLeft_F & YGLefth_R & N/A \\
\hline Plasmid Digest & BHDrN1* & KpnI & NotI & NheI \\
\hline
\end{tabular}

*Similar to previously constructed plasmid $\mathrm{BHDcN} 1^{20}$ for purposes of cloning in this study, but with the following sequence immediately 5 ' of the ApaI site sequence:

CACACTGTGTGTGCAGCTCGAGGCTCTTCCGTCAATCAAGTTCAAGGGCGACACAAAATTTATTCTAAATGCATAAT

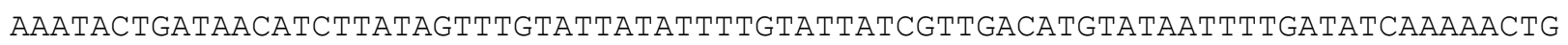

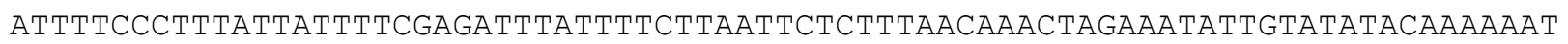
CATAAATAAAGATGAAAgTTTATTATAGGTGTTATCAATCGAAAAAGCAACGTATCTTATTTAAAGTGCGTTG СтTTTTCTCATTTATAAGGTAAATAATTCTCATATATCAAGCAAAGTGACAGGCGCCCTTAAATATTCTGACAAA TGCTCTTTCCCTAAACTCCCCCCATAAAAAAACCCGCCGAAGCGGGTTTTTACGTTATTTGCGGATTAACGATTACT CGTTATCAGAACCGCCCAGG

Right Homology Arm and gRNA:

\begin{tabular}{|l|l|l|l|}
\hline HSDygU4 & Template & Oligo/Enzyme 1 & Oligo/Enzyme 2 \\
\hline PCR Product & Genomic DNA & YGRight_F & YGRighth_R \\
\hline PCR Product & TTTygU4 & gRNA_SV40_F & gRNA_YG_R \\
\hline Plasmid Digest & HSDygU4i1 & MluI & XbaI \\
\hline
\end{tabular}

\section{Construction primers}

HSDygU4i1:

YGLeft_F: TAGGGGTCAGTGTTACAACCAATTAACCAGGTACCGTGGGTGGATTACAGGGTAGCA

YGLefth_R: TTAGTCTCTAATTGAATTAGATCCGCGGCCGCCTGCGGATGGGCTGCTCC

\section{HSDygU4:}

YGRight_F: TTTAATGTTCGCTTAATGCGTATGCATAGGCCTCCAAGGACAACAAGCCATTCG YGRighth_R: GGCATCAAACTAAGCAGAAGGCCCCTGACTCTAGAGTGGAGGGATACGGACTCAA gRNA_SV40_F: GGTTTGTCCAAACTCATCAATGTATCTTAACGCGTTTTTTTGCTCACCTGTGATTGCTC gRNA_YG_R: CCTATGCATACGCATTAAGCGAACA 
bioRxiv preprint doi: https://doi.org/10.1101/2021.05.27.446071; this version posted May 28, 2021. The copyright holder for this preprint (which

was not certified by peer review) is the author/funder, who has granted bioRxiv a license to display the preprint in perpetuity. It is made available under aCC-BY-NC 4.0 International license.

\section{TTTygU4:}

YGg_g41_F: GTGCACATAAACACGGCCAACCACAGTTTTAGAGCTAGAAATAGCAAGTTAAA
YGg_g41_R: AAAACCAGATGCAGTCCCAAGATCGTGCATCGGCCGGGAATCG
YGg_g12_F: GCACGATCTTGGGACTGCATCTGGTTTTAGAGCTAGAAATAGCAAGTTAAA
YGg_g12_R: AACTGGAGTAGTCGACCACGATGTGCACCAGCCGGGAATCG
YGg_g23_F: GCACATCGTGGTCGACTACTCCAGTTTTAGAGCTAGAAATAGCAAGTTAAA
YGg_g23_R: AACGGTCACCTCCGAGAGTCGGCTGCACCAGCCGGGAATCG
YGg_g34_F: GCAGCCGACTCTCGGAGGTGACCGTTTTAGAGCTAGAAATAGCAAGTTAAA
YGg_g34_R: TGTGGTTGGCCGTGTTTATGTGCACCAGCCGGGAATCG

Sequencing primers (for confirming plasmid sequences and sequencing resistance alleles) EGFPaLeft $S$ R: GCGAAAGCTAAGCAAATAAACAAGC

U6term_S_E : CATCTGACGTGTGTTTATTTAGAC

Yellow_gRNA1_S_F: TTGCTCACCTGTGATTGCTCC

CFD5 S R: TAGĀAATGGTTTTCCGTTGACGT

YGLeft_S_F: ACAAACGGCAAACAAACGAGG

YGLeft_S_R: TGGCGGCTAATTGAAATGTTGG

YGRight_s_E: TCGAACTGAATCAAGAGTTTGGAG

YGRight_S_R: TGAGCCACACTTCTGAGAACT 


\section{Supplementary Results}

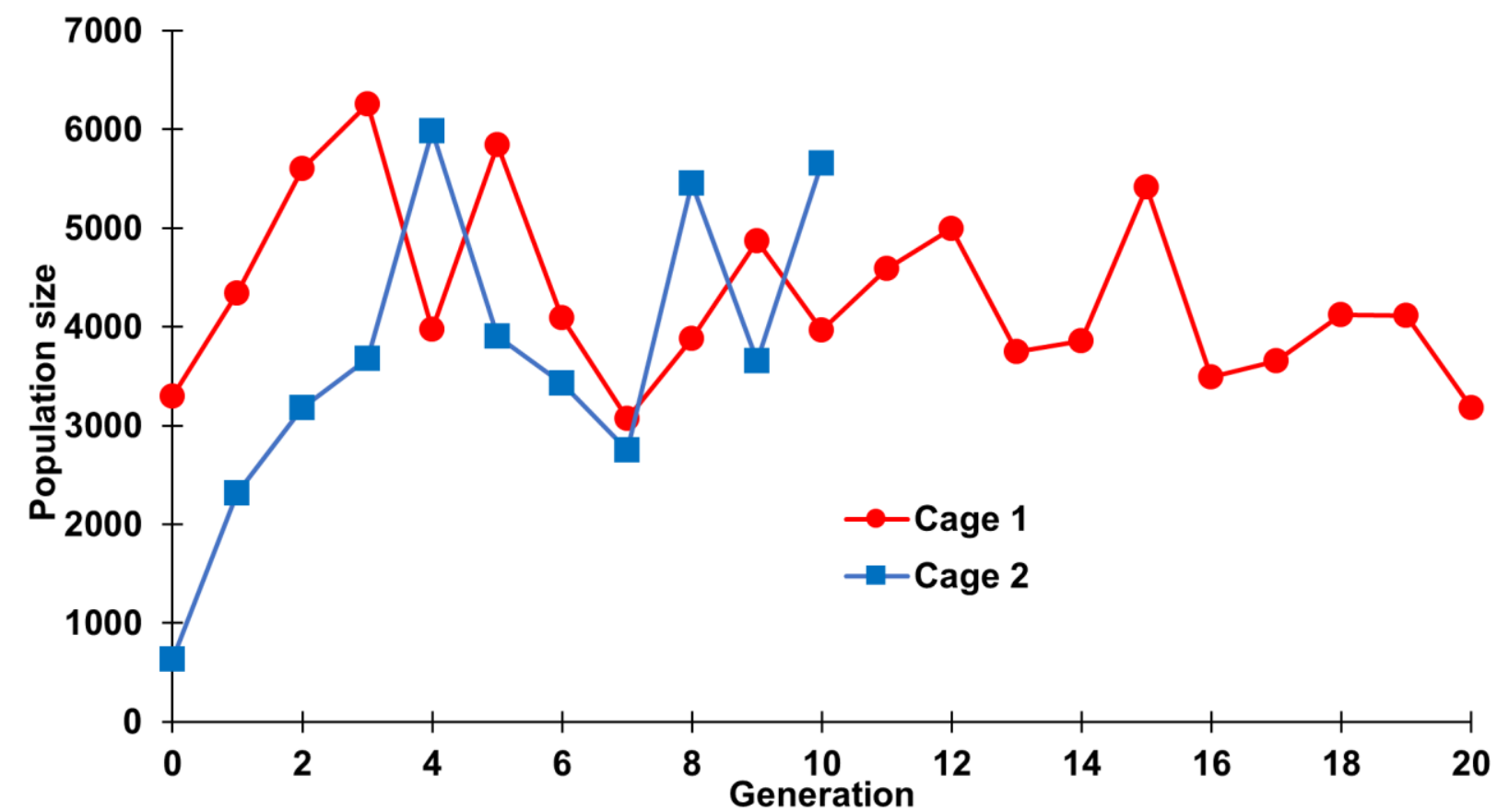

Figure S1. Cage population size. The population size for each generation is displayed for the two drive experiments cages from Figure 4. 

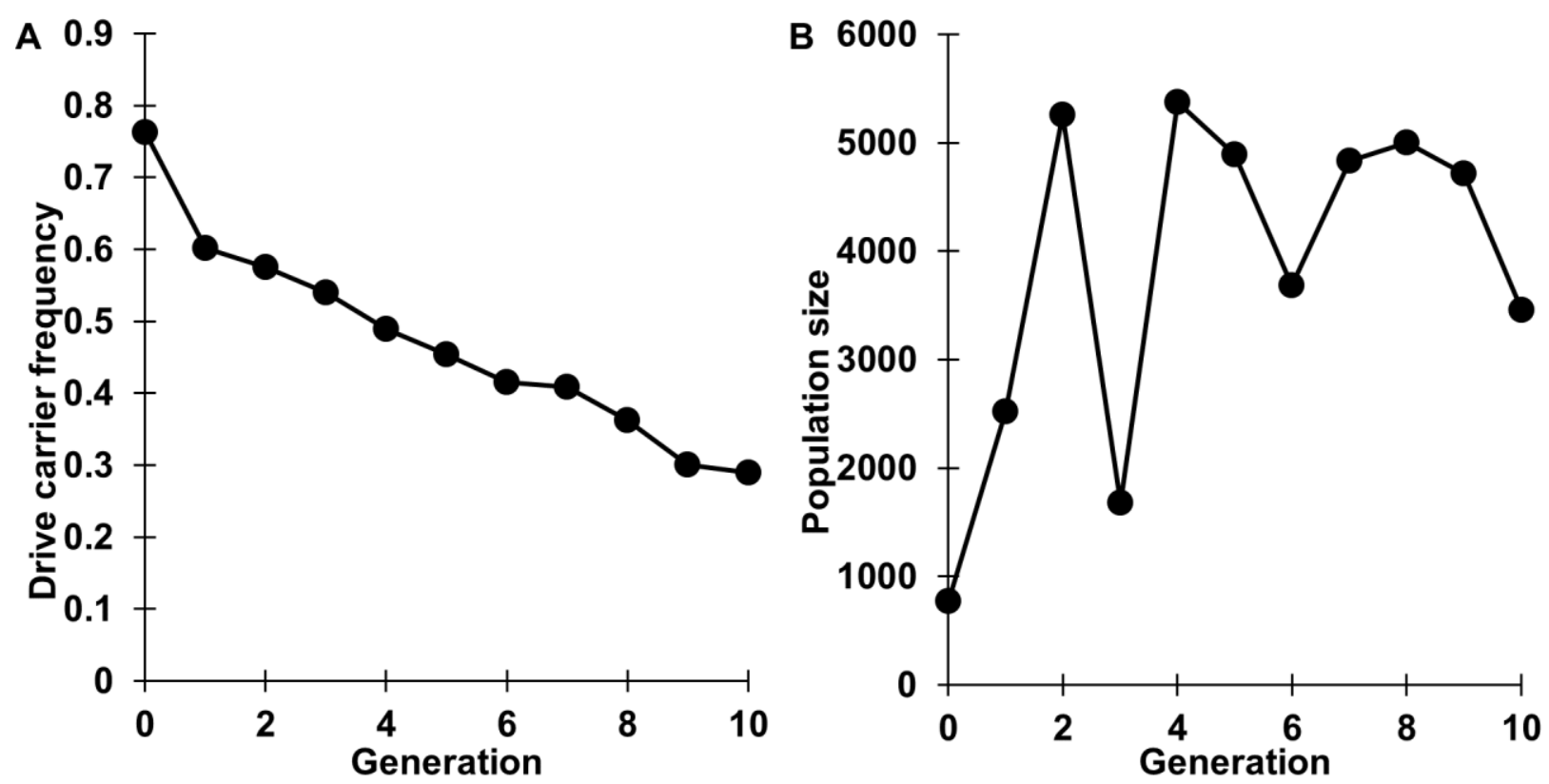

Figure S2. Cage experiment without Cas9. (A) Individuals with a drive allele and without Cas9 were introduced into a $w^{1118}$ cage population without Cas9 at a carrier frequency of $76 \%$. The cage population was followed for several non-overlapping generations, each lasting twelve days, including one day of egg-laying. All individuals from each generation were phenotyped for DsRed, with positive drive carriers having either one or two drive alleles. The drive allele cannot perform drive conversion, so it decreases in the population over time. (B) Size of the cage population in each generation. 


\section{Table S1. Maximum likelihood parameter estimates from cage populations}

Fitness values are for drive homozygotes (with multiplicative fitness per allele), except for the somatic Cas9 cleavage in females model, where the fitness parameter is applied directly to drive/wild-type females. A value of 1 is equivalent to wild-type fitness [Brackets] show 95\% confidence intervals

Log-likelihood: shows a relative probability (higher values indicate a better model fit)

AICc: Akaike information criterion, corrected (low values indicate a better match of the model without overfitting)

Table S1A. Cage 1 drive released into Cas9 background

\begin{tabular}{|l|c|c|c|c|}
\hline Fitness cost model & $\begin{array}{c}\text { Log- } \\
\text { likelihood }\end{array}$ & AICc & $\begin{array}{c}\text { Effective } \\
\text { population size }\end{array}$ & Fitness \\
\hline None & 32.0 & -61.7 & $115[57-205]$ & 1 \\
\hline $\begin{array}{l}\text { Somatic cleavage } \\
\text { female fecundity }\end{array}$ & 38.1 & -71.4 & $219[108-388]$ & $0.67[0.54-0.81]$ \\
\hline $\begin{array}{l}\text { Direct } \\
\text { fecundity and mating }\end{array}$ & 38.7 & -72.7 & $233[115-415]$ & $0.78[0.70-0.88]$ \\
\hline $\begin{array}{l}\text { Direct } \\
\text { viability }\end{array}$ & 39.0 & -73.3 & $241[118-428]$ & $0.80[0.72-0.88]$ \\
\hline $\begin{array}{l}\text { Off-target } \\
\text { fecundity and mating }\end{array}$ & 35.6 & -66.5 & $169[83-300]$ & $0.69[0.57-0.86]$ \\
\hline $\begin{array}{l}\text { Off-target } \\
\text { viability }\end{array}$ & 35.6 & -66.4 & $168[83-298]$ & $0.68[0.56-0.86]$ \\
\hline
\end{tabular}


Table S1B. Cage 2 drive released into Cas9 background

\begin{tabular}{|l|c|c|c|c|}
\hline Fitness cost model & $\begin{array}{c}\text { Log- } \\
\text { likelihood }\end{array}$ & AICc & $\begin{array}{c}\text { Effective } \\
\text { population size }\end{array}$ & Fitness \\
\hline None & 10.5 & -18.4 & $44[15-97]$ & 1 \\
\hline $\begin{array}{l}\text { Somatic cleavage } \\
\text { female fecundity }\end{array}$ & 18.0 & -30.1 & $229[77-509]$ & $0.43[0.30-0.57]$ \\
\hline $\begin{array}{l}\text { Direct } \\
\text { fecundity and mating }\end{array}$ & 15.9 & -25.9 & $143[48-319]$ & $0.63[0.51-0.77]$ \\
\hline $\begin{array}{l}\text { Direct } \\
\text { viability }\end{array}$ & 15.8 & -25.6 & $140[47-311]$ & $0.66[0.55-0.79]$ \\
\hline $\begin{array}{l}\text { Off-target } \\
\text { fecundity and mating }\end{array}$ & 16.6 & -27.2 & $166[56-370]$ & $0.53[0.42-0.67]$ \\
\hline $\begin{array}{l}\text { Off-target } \\
\text { viability }\end{array}$ & 17.1 & -28.3 & $188[63-418]$ & $0.51[0.42-0.64]$ \\
\hline
\end{tabular}

Table S1C. Drive without any Cas9 present

\begin{tabular}{|l|c|c|c|c|}
\hline Fitness cost model & $\begin{array}{c}\text { Log- } \\
\text { likelihood }\end{array}$ & AICc & $\begin{array}{c}\text { Effective } \\
\text { population size }\end{array}$ & Fitness \\
\hline None & 22.8 & $\underline{-43.0}$ & $635[214-1414]$ & 1 \\
\hline $\begin{array}{l}\text { Somatic cleavage } \\
\text { female fecundity }\end{array}$ & 22.8 & -39.5 & $635[214-1414]$ & $1.00[0.90-1.22]$ \\
\hline $\begin{array}{l}\text { Direct } \\
\text { fecundity and mating }\end{array}$ & 22.8 & -39.6 & $635[214-1414]$ & $1.00[0.93-1.17]$ \\
\hline $\begin{array}{l}\text { Off-target } \\
\text { fecundity and mating }\end{array}$ & 22.8 & -39.6 & $635[214-1414]$ & $1.00[0.94-1.15]$ \\
\hline
\end{tabular}

